\title{
Pollinator movements and the polymorphism for outcrossing rate at the ray floret locus in Groundsel, Senecio vulgaris $\mathrm{L}$.
}

\author{
Richard J. Abbott and \\ Judith A. Irwin
}

Laboratory of Plant Science, Sir Harold Mitchell Building, University of St Andrews, St Andrews, Fife, KY16 9TH, UK

The relative attractiveness to pollinators (syrphid flies) of the radiate and non-radiate morphs of Senecio vulgaris was recorded in an artificial stand in which morphs occurred at equal frequency. Pollinators showed marked discrimination in favour of the radiate morph; 72 per cent of 336 visits were to plants bearing radiate capitula. Analysis of pollinator flights between plants showed a bias for radiate to radiate transitions ( $R-R)$, followed by non-radiate to radiate ( $N-R)$, then radiate to non-radiate $(R-N)$ transitions. Flights between non-radiate plants ( $N-N)$ were very inf requent. The greater frequency of $\mathrm{N}-\mathrm{R}$ relative to $\mathrm{R}-\mathrm{N}$ transitions resulted from an increase in preference for the radiate morph as pollinators moved from the first to the second plant during a flight sequence. A cause of this behaviour is suggested. Given certain assumptions, the observed pattern of intermorph transition flights would explain, in part, the greater female outcrossing by the radiate morph based on intermorph crossing, and, in turn, the higher female outcrossing rate of radiate relative to non-radiate disc florets recorded previously in some polymorphic populations.

\section{INTRODUCTION}

In populations of Senecio vulgaris (Compositae) that are polymorphic for capitulum type, radiate plants exhibit significantly greater female outcrossing rates than non-radiate plants (Marshall and Abbott, 1982, 1984a). This conclusion is based on estimates of outcrossing between morphs; outcrossing rates within morphs have yet to be determined. In one of two populations subjected to detailed study by Marshall and Abbott (1984b), the greater female outcrossing of radiate relative to non-radiate plants was entirely accounted for by the higher outcrossing of pistillate (female) ray florets in radiate capitula; in the same capitula, hermaphrodite disc florets outcrossed at rates equivalent to those recorded for disc florets of non-radiate plants. The greater female outcrossing of ray florets was attributed by Marshall and Abbott $(1984 b)$ to the occurrence of functional protogyny in radiate capitula (see also Burtt, 1977): However, Warren et al. (1987) have recently shown that some radiate plants produce self-incompatible ray florets, in addition to self-compatible disc florets, and this ensures outcrossing in the ray floret fraction of these plants. In the second population subjected to detailed study by Marshall and Abbott
$(1984 b)$, the difference between morphs in outcrossing rates was only partly explained by the greater outcrossing of ray relative to disc florets. In this second population it was apparent that the disc florets of radiate plants also exhibited rates of female outcrossing far in excess of those shown by the equivalent florets of non-radiate plants. Marshall and Abbott (1984b) advanced two possible causes for the higher outcrossing rates of disc florets of radiate relative to non-radiate plants: (a) disc florets of radiate plants may exhibit some form of cryptic self-incompatibility mechanism that inhibits the germination and/or pollen tube growth of self-pollen; (b) in polymorphic populations radiate plants are more attractive to pollinators and one effect of this is to cause pollinators to move between morphs more frequently than expected in the non-radiate to radiate direction than in the opposite direction thus boosting the female outcrossing rate of radiate plants based on intermorph crosses. Warren et al. (1987) have since produced evidence which suggests that there may be no difference between morphs in the expression of cryptic self-incompatibility in disc florets. Here we report a study which shows that in polymorphic stands radiate plants are considerably more attractive to pollinators and, moreover, pollinators are 
biased in their movements between morphs with transition flights from non-radiate to radiate plants occurring more frequently than in the opposite direction.

\section{MATERIALS AND METHODS}

An artificial stand containing 18 radiate and 18 non-radiate potted plants of $S$. vulgaris arranged alternately in a $6 \times 6$ rectangular grid with a spacing of one metre between adjacent plants was set out in a disused airfield at Crail, Fife. Plants had been raised from seed originally collected from a polymorphic population at Newhaven Road, Edinburgh (Grid Ref. NT 258758). On three different days the flight patterns of all insect pollinators (syrphid flies) entering the stand were monitored over a 4-hour period (approximately 11.00 a.m. to 3.00 p.m.). Syrphid flies are the common pollinators of $S$. vulgaris in Scotland, and were visiting groundsel and other composite species already growing at the airfield. Each day before monitoring commenced, a count was made of the total number of open radiate and non-radiate capitula within the plot.

\section{RESULTS AND DISCUSSION}

The results from the mixed stand show that each day pollinators discriminated in favour of the radiate type (table 1). Overall pollinators made 72 per cent of their visits to radiate plants $(N=336$ visits, $\left.\chi_{(1)}^{2}=65 \cdot 19, P<0 \cdot 001\right)$. Even greater was the bias towards capitulum type with pollinators tending to forage more radiate than non-radiate capitula during visits to the respective plants. The mean number of capitula foraged by a pollinator during individual visits to radiate or non-radiate plants was respectively 1.93 and $1.45,1.71$ and 1.33 , and 2.35 and 1.72 over the three days that records were taken.

Analysis of pollinator flights between plants (transitions) (table 2) showed that on the 2 days when pollinators were most active there was a distinctive preference for radiate to radiate $(\mathrm{R}-\mathrm{R})$ flights followed by non-radiate to radiate $(N-R)$, then radiate to non-radiate $(\mathrm{R}-\mathrm{N})$ transitions. Transition flights between non-radiate plants $(\mathrm{N}$ $\mathrm{N}$ ) were very infrequent. If it is assumed that pollen production of morphs is equivalent (as recorded in some natural populations for plants that produce equal numbers of capitula, see Ross and Abbott, 1987), and pollen pick-up, retention and carry-over by pollinators is the same for each morph, then the observed pattern of intermorph transition flights would lead to a greater female outcrossing by the radiate morph based on intermorph outcrossing. This, in turn, would explain, at least in part, the higher outcrossing rate of radiate relative to non-radiate disc florets recorded previously in some polymorphic populations (Marshall and Abbott, 1984b). The greater frequency of $\mathrm{N}-\mathrm{R}$ transitions relative to $\mathrm{R}-\mathrm{N}$ transitions does at first seem a somewhat surprising

Table 1 Observed frequencies of pollinator visits to (i) plants, and (ii) capitula, of the radiate and non-radiate morphs of Senecio vulgaris in mixed stands

\begin{tabular}{|c|c|c|c|c|}
\hline & Morph & $27: 5: 85$ & $\begin{array}{c}\text { Dat } \\
28: 5: 85\end{array}$ & $3: 6: 85$ \\
\hline \multicolumn{5}{|l|}{ (a) Plants } \\
\hline & Radiate & 0.772 & 0.667 & 0.695 \\
\hline & Non-radiate & $0 \cdot 228$ & 0.333 & $0 \cdot 305$ \\
\hline & $x_{(1)}^{2} \dagger$ & $40 \cdot 26^{* * *}$ & $8 \cdot 00^{* *}$ & $19 \cdot 53 * *$ \\
\hline & $\mathrm{N}_{1}$ & 136 & 72 & 128 \\
\hline \multicolumn{5}{|l|}{ (b) Capitula } \\
\hline & Radiate & $0 \cdot 818$ & 0.719 & 0.757 \\
\hline & Non-radiate & $0 \cdot 182$ & $0 \cdot 281$ & 0.243 \\
\hline & $x_{(1)}^{2} \neq$ & $108 \cdot 92^{* * *}$ & $24 \cdot 13^{* * *}$ & $76 \cdot 93^{* * *}$ \\
\hline & $\mathrm{N}_{2}$ & 248 & 114 & 276 \\
\hline
\end{tabular}

$\dagger \chi^{2}$ is for the null hypothesis of equal visitation to radiate and non-radiate plants, and $\ddagger \chi^{2}$ is for the null hypothesis of visitation based on the relative frequencies of capitulum types in stands. (The total number of open radiate and non-radiate capitula in the plot on each of the three dates was respectively 363 and 345,334 and 320 , and 341 and 332). $N_{1}$ and $N_{2}$ equal the number of pollinator visits to plants and capitula respectively. The number of pollinators which visited the stand each day was 66,46 and 57 on the three respective dates ${ }^{* *} P<0 \cdot 01$, *** $P<0 \cdot 001$ ). 
Table 2 Transition frequencies for pollinator flights between plants in mixed stands

\begin{tabular}{lccc}
\hline & \multicolumn{1}{c}{ Date } \\
Transition & $27: 5: 85$ & $28: 5: 85$ & $3: 6: 85$ \\
\hline R-R & 0.543 & $0 \cdot 346$ & 0.409 \\
N-R & $0 \cdot 314$ & $0 \cdot 346$ & 0.338 \\
R-N & $0 \cdot 129$ & 0.308 & $0 \cdot 211$ \\
N-N & 0.014 & 0.000 & 0.042 \\
$\chi_{(3)}^{2}$ & $44 \cdot 86^{* * *}$ & $8 \cdot 77^{*}$ & $22 \cdot 01^{* * *}$ \\
$N$ & 70 & 26 & 71
\end{tabular}

Transitions recorded were between radiate to radiate $(R-R)$, non-radiate to radiate $(\mathrm{N}-\mathrm{R})$, radiate to non-radiate $(\mathrm{R}-\mathrm{N})$ and non-radiate to non-radiate $(\mathrm{N}-\mathrm{N})$ plants. $\chi^{2}$ was for the null hypothesis of equal transition frequencies. $N$ equals the number of pollinator transition flights.

result. Simple preference for the radiate morph held at constant level throughout the period a pollinator is foraging in a mixed stand would not in itself lead to a higher frequency of intermorph transitions in one direction rather than the other. However, if the preference for the radiate type increases as a pollinator moves from the initial to subsequent plants during a flight sequence then a bias will occur in the direction that was found. This, in fact, is what happened on days 1 and 3 in the mixed stand (table 3 ). On both of these days pollinator preference for the radiate morph was substantially greater for the second plant than for the first plant visited during a flight sequence. Thereafter, preference for the radiate morph decreased over the third and fourth plants visited though tended to remain at a higher level than was recorded for the first plant chosen. (N.B. Radiate preference values for third and fourth plants have relatively large standard errors due to the small number of pollinators that visited these plants over all flight sequences [table 3].) Taken overall we may conclude that the bias towards N-R transitions observed in the present study stemmed mainly from an increase in preference for the radi- ate type as pollinators moved from the initial to the second plant during a flight sequence. This form of bias will be maximised, when pollinators visit very few plants in a plot as was the case in the present study where mean number of plants visited per pollinator was respectively $2.06,1.56$ and 2.25 on each of the three days that records were taken. It remains to be established why the preference of a pollinator for the radiate morph is greater for the second than the first plant visited during a flight sequence. One possibility is that syrphid flies, which visit $S$. vulgaris to collect pollen, find that non-radiate plants offer poor rewards for the energy expended in extracting pollen from capitula. If this is so, then a pollinator that initially visits a non-radiate morph may tend to switch quickly to the more attractive radiate morph as it moves to a second plant in a mixed stand. In contrast, if rewards from radiate plants are sufficient to satisfy the demands of a syrphid fly, because, for example, radiate capitula are more easily manipulated for pollen extraction, then a fly is likely to maintain its preference for the radiate morph when moving to a second plant in the stand. Clearly an analysis of the value and ease with which rewards are obtained by pollinators from each morph is now required to test this hypothesis.

A final point to emerge from the foraging patterns of pollinators observed in the present study is that most outcrossing in a polymorphic population is expected to occur between plants of the radiate morph while outcrossing between nonradiate plants should be negligible. The flight patterns of pollinators in polymorphic populations should, therefore, favour the radiate morph as both a recipient and donor of cross pollen. A consequence of this is that the radiate morph will be expected to show a significantly greater level of male outcrossing as well as female outcrossing compared with the non-radiate morph. Clearly the complex spatial arrangement of morphs together with frequency and density variation of plants in

Table 3 Observed frequencies of pollinator visits to the radiate morph recorded for the initial and subsequent plants visited over all flight sequences

\begin{tabular}{lllll}
\hline & 1 & \multicolumn{3}{c}{ Order of plants visited during a flight sequence } \\
\hline Day 1 $(27: 5: 85)$ & $0.682(66)$ & $0.909(44)$ & $0.722(18)$ & $0.833(6)$ \\
Day 2 $(28: 5: 85)$ & $0.652(46)$ & $0.684(19)$ & $0.667(6)$ & $1.000(1)$ \\
Day 3 $(3: 6: 85)$ & $0.632(57)$ & $0.762(42)$ & $0.739(23)$ & $0.600(5)$ \\
Over all days $\dagger$ & $0.657 \pm 0.036(169)$ & $0.809 \pm 0.038(105)$ & $0.723 \pm 0.065(47)$ & $0.750 \pm 0.125(12)$ \\
\hline
\end{tabular}

$\uparrow$ Standard errors attached

Figures in parentheses show number of pollinators that visited 1,2,3,4 or 5 different plants over all flight sequences 
natural polymorphic populations is likely to affect greatly the actual levels of male and female outcrossing shown by each morph. Investigations aimed at determining the possible effects of these additional factors are now required.

Acknowledgments We are grateful to an anonymous referee for helpful comments on an earlier draft of the manuscript.

\section{REFERENCES}

BURTT, B. L. 1977. Aspects of diversification in the capitulum. Heywood, V. H., Harborne, J. B. and Turner, B. L. In The Biology and Chemistry of the Compositae Vol. 1, Academic Press, London, pp. 41-59.
MARSHALL, D. F. AND ABBOTT, R. J. 1982. Polymorphism for outcrossing frequency at the ray floret locus in Senecio vulgaris L. I. Evidence. Heredity, 48, 227-235.

MARShALL D. F. AND ABBOTT, R. J. $1984 a$ Polymorphism for outcrossing frequency at the ray floret locus in Senecio vulgaris L. II. Confirmation. Heredity, 52, 331-336.

MARShALL, D. F. AND ABBOTT, R. J. 1984 b. Polymorphism for outcrossing frequency at the ray floret locus in Senecio vulgaris L. III. Causes. Heredity, 53, 145-149.

ROSS, M. D. AND ABBOTT, R. J. 1987. Fitness, sexual asymmetry, functional sex and selfing in Senecio vulgaris L. Evolutionary Trends in Plants, 1, 21-28.

WARREN, J. M., CRAWFORD, T. J. AND OXFORD, G. S. 1987. Inhibition of self-pollen germination in Senecio vulgaris $\mathrm{L}$. Heredity, (in press). 